\title{
UNA RUTA TURÍSTICA CAMINO AL DESARROLLO LOCAL DE LA PARROQUIA
} LA PILA

AUTORES: $\quad$ Shirley Pamela Gómez Bailón ${ }^{1}$

Luis Fernando Lucio Villacreses ${ }^{2}$

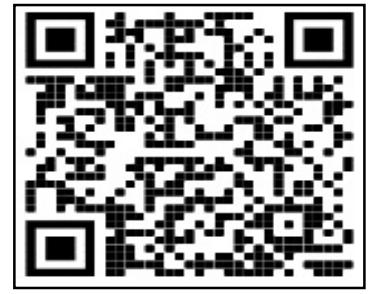

DIRECCIÓN PARA CORRESPONDENCIA: gomez-shirley8904@unesum.edu.ec

Fecha de recepción: 19/08/2020

Fecha de aceptación: 26/11/2020

\section{RESUMEN}

El turismo es una actividad económica que ha obtenido un crecimiento considerable, y su aplicación resulta eficaz como una estrategia de innovación y desarrollo para localidades que tienen potencialidad turística, con ello es notorio considerar la necesidad de una planificación desde una perspectiva territorial, es así, que el presente artículo muestra como una ruta turística es considerada una estrategia para el desarrollo local, además de la percepción ciudadana captada mediante una encuesta, con el objetivo de analizar su aporte en la parroquia, conforme a ello, se interpretaron los datos obtenidos y se detallan gráficos que representan la opinión de los habitante contrastada con situación actual según el PDOT del 2015 y la información obtenida del Instituto Nacional de Estadísticas y Censo (INEC) 2010, estableciendo una metodología cuantitativa y cualitativa para detallar los resultados, en efecto, la principal actividad dentro de la parroquia es el turismo y por su parte, se propone la creación una ruta turista que ofrece importantes oportunidades de desarrollo sostenible y por lo tanto los Objetivos de Desarrollo Sostenible (ODS) se posicionan en su creación, haciendo partícipe a todos los habitantes y se enfatiza el rol que desempeña en el territorio para contribuir a la construcción del progreso de las diferentes actividades, enfocadas y vinculadas entre aspectos ambientales, todo ello orientado al mejoramiento de la calidad de vida a partir de su creación, por ello, existe interés de parte del gobierno parroquial de implementar la ruta turística y la opinión ciudadana es punto importante a destacar, de acuerdo con los resultados de la encuesta, esta corrobora que participaría en ella, lo que permite que se cumpla con objetivos de desarrollo sostenible considerando su planificación para su posterior ejecución.

\footnotetext{
${ }^{1}$ Estudiante de la carrera de Ingeniería Ambiental de la Universidad Estatal del Sur de Manabí. Jipijapa, Manabí, Ecuador. gomez-shirley8904@unesum.edu.ec ORCID ID: https://orcid.org/0000-0001-7032-6223

${ }^{2}$ Ing. Forestal, Magister en Educación y Desarrollo Social, Docente de la Carrera de Ingeniería Ambiental de la Universidad Estatal del Sur de Manabí. Jipijapa, Manabí, Ecuador. luis.lucio@unesum.edu.ec ORCID ID: https://orcid.org/0000-0002-3757-7183
}

(C) Universidad Estatal del Sur de Manabí. Jipijapa, Ecuador. 
PALABRAS CLAVE: turismo rural comunitario; acciones sociales; producción sostenible.

\title{
A TOURIST ROUTE WAY TO THE LOCAL DEVELOPMENT OF LA PILA PARISH
}

\begin{abstract}
Tourism is an economic activity that has obtained considerable growth, and its application is effective as an innovation and development strategy for localities that have tourist potential, with this it is notorious to consider the need for planning from a territorial perspective, it is thus, that this article shows how a tourist route is considered a strategy for local development, in addition to the citizen perception captured through a survey, with the aim of analyzing its contribution in the parish, accordingly, the data obtained were interpreted and detail graphics that represent the opinion of the inhabitants contrasted with the current situation according to the PDOT of 2015 and the information obtained from the INEC 2010 census, establishing a quantitative and qualitative methodology to detail the results, in effect, the main activity within the parish is the tourism and for its part, it is proposed to create a tourist route that offers There are important opportunities for sustainable development and therefore the SDGs are positioned in their creation, involving all the inhabitants and emphasizing the role they play in the territory to contribute to the construction of the progress of the different activities, focused on and linked between environmental aspects, all aimed at improving the quality of life from its creation, therefore, there is interest on the part of the parish government to implement the tourist route and citizen opinion is an important point to highlight, according to the results of The survey confirms that it would participate in it, which allows it to meet sustainable development objectives considering its planning for subsequent execution.
\end{abstract}

KEYWORDS: rural community tourism; social actions; sustainable production

\section{INTRODUCCIÓN}

En los últimos años, el turismo se ha convertido en un factor fundamental de desarrollo socioeconómico (Kotler, 1997), habiéndose otorgado a escala mundial, mucha importancia al desarrollo de distintas actividades relacionadas con el ocio y la recreación, con miras a satisfacer las crecientes e innovadoras demandas de los turistas. El desarrollo local como una aproximación es un concepto que tiene actualmente una enorme vigencia y engloba diferentes corrientes de pensamiento. Desde las posturas que lo reivindican como una estrategia a partir de la cual, las ciudades pueden posicionarse en el contexto global para captar inversiones extranjeras como un modelo de crecimiento exógeno (Varisco, 2008).

A su vez la búsqueda de una teoría que enmarque las actividades de intervención y mediación del desarrollo es fundamental; significa pasar de una concepción tradicional del desarrollo a una nueva visión, como algo construido a partir de las capacidades de los actores locales (Cara, s. f.).

En su concepción, este modelo tiene consecuencias sobre la participación política, las necesidades sociales y económicas, en particular, el nivel de empleo, la creación del valor a partir de recursos locales, la formación de capital social inclusive capital humano y la sostenibilidad del 
medio ambiente (Gambarota \& Lorda, 2017). En esta medida, se reconoce al territorio como una construcción social, que está formada por procesos que se desarrollan en el tiempo y espacio, que resulta de la incidencia de relaciones con diferentes lógicas, adoptadas por los distintos actores, a fin de lograr su conformación. El territorio es un factor de transformación, donde los actores se desarrollan interrelacionándose, lo que permite observar con claridad la potencialidad que posee, y entender a este como un ente que se nutre de las acciones de sus instituciones y gobernantes para lograr el incremento de su competitividad.

Así, el turismo sostenible es de mayor importancia y tiene como tarea constituirse en una estrategia capaz de salvaguardar y respetar el patrimonio natural y cultural, promover y fortalecer el desarrollo económico local y por sobre todo fomentar e integrar la participación de actores locales en su modelo de gestión. Es por esto, que debe ser considerada como una estrategia de desarrollo local y entregar los lineamientos generales a la amplia oferta turística disponible y transformarse en el gran plan maestro que guíe el desarrollo turístico en sus distintas escalas (Periódico Verde, 2011).

El propósito del desarrollo local turístico, destaca Abellán et al., (2008), que busca el mejoramiento de las condiciones de vida de una determinada localidad, de tal manera, es una actividad que es reconocida como motor de desarrollo, y se otorgará un mayor interés a la creación de rutas turísticas, realizando previo análisis de los recursos con los que cuenta una determinada área para poder determinar su potencialidad.

De esta manera, se proporcionan alternativas de desarrollo territorial, a través de las líneas que enmarca el turismo, en este contexto, la actividad turística impactará positivamente en su desarrollo económico y en la calidad de vida de las personas, si bien puede generar aspectos negativos que influirán directamente en la población local como una alta dependencia de la actividad turística, aumento de precios, degradación del espacio y una posible contaminación, los cuales con una adecuada planificación pueden atenuarse o evitarse. Tal como afirma Vera et al. (1997) La creciente demanda de destinos y experiencias alternativas ha propiciado que todos los espacios sean potencialmente turísticos, en razón a su especificidad, y agrega que el turismo debe ser reconocido como pieza en la construcción de nuevos espacios de crecimiento a escala local en la transformación de las sociedades y territorios. El turismo sostenible, atiende a las necesidades de los turistas actuales y de los receptores, mientras que al mismo tiempo protege y fomenta las oportunidades para el futuro. Se concibe así, como una vía hacia la gestión de los recursos de manera que puedan satisfacer las necesidades económicas, sociales y estéticas, respetando la integridad cultural, los procesos ecológicos, la diversidad biológica y los sistemas que sostienen la vida.

El objetivo de la presente investigación es analizar el aporte al desarrollo local que generaría una ruta turística en la parroquia La Pila y para ello se interpretó los datos de una encuesta realizada a la ciudadanía. Además de determinar los principales aportes que se surgen en la parroquia por la presente.

(C) Universidad Estatal del Sur de Manabí. Jipijapa, Ecuador. 


\section{DESARROLLO}

En el desarrollo de la presente investigación se utilizó materiales de consulta digital que contenían información relevante a la temática abordada, además se empleó la técnica de encuesta, que es ampliamente utilizada como procedimiento de investigación, puesto que, permite obtener y elaborar datos de modo rápido y eficaz.

Se puede definir la encuesta, siguiendo a García Ferrando, como una técnica que utiliza un conjunto de procedimientos estandarizados de investigación mediante los cuales se recoge y analiza una serie de datos de una muestra de casos representativa de una población o universo más amplio, del que se pretende explorar, describir, predecir y/o explicar una serie de características. Para Sierra Bravo, la observación por encuesta, que consiste igualmente en la obtención de datos de interés sociológico mediante la interrogación a los miembros de la sociedad, es el procedimiento sociológico de investigación más importante y el más empleado. Entre sus características se pueden destacar las siguientes:

1. La información se obtiene mediante una observación indirecta de los hechos, a través de las manifestaciones realizadas por los encuestados, por lo que cabe la posibilidad de que la información obtenida no siempre refleje la realidad.

2. La encuesta permite aplicaciones masivas, que mediante técnicas de muestreo adecuadas pueden hacer extensivos los resultados a comunidades enteras.

3. El interés del investigador no es el sujeto concreto que contesta el cuestionario, sino la población a la que pertenece; de ahí, como se ha mencionado, la necesidad de utilizar técnicas de muestreo apropiadas.

4. Permite la obtención de datos sobre una gran variedad de temas.

5. La información se recoge de modo estandarizado mediante un cuestionario (instrucciones iguales para todos los sujetos, idéntica formulación de las preguntas, etc.), lo que faculta hacer comparaciones inter grupales (García 1993).

Los métodos utilizados en el presente estudio se los detalla a continuación:

\section{Método Cualitativo}

En sentido amplio, pude definirse la metodología cualitativa como la investigación que produce datos descriptivos que trata de identificar la naturaleza profunda de las realidades, su sistema de relaciones, su estructura dinámica.

Básicamente esta comienza con la recolección de datos, mediante la observación empírica o mediciones de alguna clase, y a continuación se construye, a partir de las relaciones descubiertas, sus categorías y proposiciones teóricas. Pretenden descubrir una teoría que justifique los datos. Mediante el estudio de los fenómenos semejantes y diferentes analizados, desarrolla una teoría explicativa. 
Se centra en el descubrimiento de constructos y proposiciones a partir de una base de datos o fuentes de evidencia (observación, entrevista, documentos escritos). A partir de los datos, que se ordenan y clasifican, se generan constructos y categorías.

Se orienta al descubrimiento de los constructos analíticos o categorías que pueden obtenerse a partir del continuo comportamental; es un proceso de abstracción en el que las unidades de análisis se revelan en el transcurso de la observación y descripción. Mediante estrategias adecuadas, se busca obtener y analizar datos de tipo subjetivo, su propósito es reconstruir las categorías específicas que los participantes emplean en la conceptualización de sus experiencias y en sus concepciones (Lecanda \& Garrido, s. f.).

\section{Método Cuantitativo}

Rodríguez Peñuelas (2010) señala, que el método cuantitativo se centra en los hechos o causas del fenómeno social, con escaso interés por los estados subjetivos del individuo. Comienza con un sistema teórico, desarrolla definiciones operacionales de las proposiciones y conceptos de la teoría y las aplica empíricamente en algún conjunto de datos. Pretenden encontrar datos que ratifiquen una teoría. No solo intenta determinar la medida en que se cumple una proposición, probar empíricamente que una hipótesis dada es aplicable a varios conjuntos de datos, sino que también procura establecer generalizaciones con relación al universo de poblaciones al que ésta es aplicable.

Es un proceso en el que las unidades de análisis, previamente derivadas o definidas son sometidas a un cómputo o enumeración sistemáticos. Aplica categorías conceptuales y relaciones explicativas aportadas por observadores externos al análisis específico de las poblaciones concretas. Determina la medida en que la conducta real de los participantes se corresponde con las categorías y relaciones explicativas aportadas por los observadores externos (López, 2018).

La Pila es una parroquia rural del cantón Montecristi, caracterizada por reflejar las costumbres y tradiciones de las culturas que se desarrollaron en la provincia de Manabí, y por ser elaboradas en tiempos prehispánicos. Consta de un manantial de agua medicinal azufrada con muchos minerales y tiene forma de cono invertido, en donde se encuentran muchos vestigios arqueológicos, plata, bronce y láminas de oro.

Esta parroquia tiene 2.452 habitantes de acuerdo a la información proporcionada por el Instituto Nacional de Estadísticas y Censos del año 2010, así, la población más sobresaliente es la masculina con el 52,45\% mientras que la femenina es el 47,55\%. La tasa de Analfabetismo es $7,97 \%$ considerado un porcentaje alto y la tasa de crecimiento poblacional ha decrecido en el último periodo a un ritmo de 1,88\% del promedio anual y el $0,49 \%$ de la población total emigraron por escasa plazas de trabajo.

Actualmente la tendencia de la población es la actividad artesanal, es necesario concientizar a la población en su identidad cultural, fortalecer sus costumbres y tradiciones ya que esto permitirá

(C) Universidad Estatal del Sur de Manabí. Jipijapa, Ecuador. 
mantener sus raíces y saberes ancestrales logrando ser permanente a pesar del tiempo y trascienda de generación en generación.

\section{Proyección de la población de la parroquia La Pila}

Las proyecciones son fundamentales para llevar un control desde la realización del último censo hasta que se realice el siguiente, así los métodos matemáticos y estadísticos son una herramienta para las tendencias de las variables que componen la ecuación compensadora obteniendo un abanico de escenarios futuros posibles.

Los métodos en general no son precisos, considerando la variable población el logarítmico fue el de mayor utilidad y precisión por su tendencia al crecimiento demográfico en la parroquia La Pila.

Tabla 1. Datos obtenidos a partir de proyección logarítmica de datos.

\begin{tabular}{ll}
\hline AÑO & POBLACIÓN \\
\hline 1990 & 1.756 \\
2001 & 2.071 \\
2010 & 2.452 \\
2020 & 2.400 \\
2030 & 2.577 \\
2040 & 2.714 \\
2050 & 2.826 \\
\hline
\end{tabular}

El procedimiento de este método se basa en la determinación de la población con los censos conocidos, con la aplicación de una fórmula, para posteriormente realizar la proyección de población para los años requeridos.

\section{El territorio como resultado de las acciones sociales}

El territorio es considerado como un sistema constituido por varios subsistemas (natural, social, cultural, tecnológico, productivo, político), interactivo y dinámico, y se traduce en la consideración de varios factores del desarrollo, que pueden sintetizarse en infraestructuras, mercado de trabajo, recursos ambientales, estructura productiva y espacial (Mendez 1997). Considerando que el espacio socialmente construido, puede apoyar u obstaculizar los procesos de desarrollo, este se basa en una adecuada utilización de los recursos propios y orientarse a satisfacer las necesidades de la población. De esta manera, las medidas de apoyo más adecuadas, serán las destinadas a revalorizar esas potencialidades territoriales, y promover iniciativas que puedan utilizar los grupos humanos en forma eficiente. Todo lo expuesto, exige poseer un adecuado conocimiento de la realidad local, siendo aconsejable una descentralización de las

182 UNESUM-Ciencias. Publicación cuatrimestral. Vol. 4, No. 4 (Septiembre-Diciembre), Año 2020. 
decisiones políticas, lo cual facilita la definición de objetivos apropiados para cada caso y una gestión más eficaz.

Siguiendo la teoría del desarrollo local, el territorio puede considerarse como actor, y no solamente como un conjunto de recursos naturales o infraestructura, siempre que se lo entienda como una compleja trama de relaciones que los individuos producen en su interacción determinando una identidad local. Los turistas provienen por definición de otros centros emisores, y los residentes conforman la comunidad anfitriona; los otros subsistemas pueden tener su centro de actividad e interés tanto en el territorio como fuera de él (Gambarota \& Lorda, 2017).

\section{Desarrollo turístico sostenible}

El desarrollo turístico puede definirse específicamente como la provisión y el mejoramiento de las instalaciones y servicios idóneos, y definido de una manera más general, puede también incluir los efectos asociados, tales como la creación de empleos o la generación de ingresos” (Proaño Lucero, s. f.). Una característica esencial del turismo, es que su desarrollo no se presenta de manera homogénea en el espacio, sino que genera concentraciones de la actividad, siempre en relación a la materia prima del turismo (Varisco, 2008). El turismo sostenible, para Schulte (2003), atiende a las necesidades de los turistas actuales y de las regiones receptoras, mientras que al mismo tiempo protege y fomenta las oportunidades para el futuro. Se concibe así, como una vía hacia la gestión de los recursos de manera que puedan satisfacer las necesidades económicas, sociales y estéticas, respetando la integridad cultural, los procesos ecológicos, la diversidad biológica y los sistemas que sostienen la vida.

La sostenibilidad, según Xercavins, Cayuela, Cervantes \& Sabater (2005), es gestionar y conservar la base de los recursos naturales, orientando los cambios institucionales y tecnológicos de tal manera de asegurar la capacidad de satisfacción continuada de las necesidades de las presentes y futuras generaciones. Asimismo, sostienen los citados autores que la sostenibilidad también es el aprovechamiento de los recursos naturales - culturales, con la característica que ese uso de recursos se fundamenta en la preservación y conservación de los mismos, de esta forma se evita la degradación de la zona. De allí que para lograr la sostenibilidad en el desarrollo local en un determinado territorio, se deberá mantener un equilibrio en el ámbito económico, social y ambiental, los cuales se entrelazan en todas las acciones que se ejecuten dentro de la planificación del territorio, con la finalidad de fortalecer el desarrollo local.

El Turismo Sostenible tiene como tarea constituirse en una estrategia capaz de salvaguardar y respetar el patrimonio natural y cultural, promover y fortalecer el desarrollo económico local y por sobre todo fomentar e integrar la participación de actores locales en su modelo de gestión. Es por esto que debe ser considerada como una estrategia de desarrollo local y entregar los lineamientos generales a la amplia oferta turística disponible y transformarse en el gran plan maestro que guíe el desarrollo turístico en sus distintas escalas (Periódico Verde, 2011).

(C) Universidad Estatal del Sur de Manabí. Jipijapa, Ecuador. 


\section{El turismo como estrategia de desarrollo local}

El turismo es una actividad que en la actualidad es reconocida como motor de desarrollo en un territorio provocando que los distintos gobiernos y entidades privadas, le otorguen una mayor atención promoviendo diferentes programas y actividades de fomento a la misma. Más aún puede convertirse en una estrategia de desarrollo local teniendo en consideración que se amplían cada vez más, las modalidades turísticas, ajustándose a las necesidades de los consumidores.

El desarrollo local, no sólo puede ser comprendido desde una perspectiva económica, sino lo contrario, ya que es un proceso dinámico de ampliación de capacidades locales que permita trabajar en mejorar la calidad de la vida de todos los integrantes de la población. Rescatando a Coraggio, podemos mencionar que el objetivo del desarrollo local tiene cuatro componentes básicos que son:

Económicos: Hace énfasis en el trabajo productivo, ingreso, satisfacción racional de necesidades legítimas, suficiencia y calidad de bienes públicos.

$>$ Sociales: Integración de condiciones de creciente igualdad, efectiva igualdad de oportunidades, convivencia, justicia social.

> Culturales: Pertenencia e identidad histórica, integración a comunidades con contención, valores como la solidaridad como valor moral pero también un componente funcional (nadie puede vivir mejor si su entorno no mejora sensiblemente de manera generalizada).

> Políticos: Transparencia, legitimidad y responsabilidad de las representaciones, participación directa responsable e informada de la ciudadanía en las grandes decisiones colectivas y en la gestión pública (Coragio, 2006)

En tal sentido, entendemos que el término "local” no es algo pequeño, si no es la condición común de una población que comparte una historia de asentamiento, una vida cotidiana cara a cara con distintos matices más o menos desiguales, problemáticos, con sentido solidario de reciprocidad en un espacio territorial que está conectado con otras localidades, regiones hasta llegar a lo nacional.

El desarrollo local y el turismo son a la vez estrategias de desarrollo que están en función del potencial territorial y de la riqueza natural, cultural y social que representa una estrategia diferente para el desarrollo. Lo cierto es que se extiende la brecha entre ricos y pobres, y que el número de pobres es el más grande de su historia y el acceso a los servicios es muy restringido en salud, educación y empleo. Bajo aquello, se considera al turismo como una estrategia local que permite proyectar el desarrollo desde una óptica de preservación del medio ambiente y riqueza cultural social como estrategia de vida comunitaria.

\section{Aportes del turismo al desarrollo local}

Considerando al turismo como una estrategia al desarrollo se consideran los siguientes aportes como beneficio para la sociedad y el territorio.

Mejora la inversión y la infraestructura física y tecnológica.

184 UNESUM-Ciencias. Publicación cuatrimestral. Vol. 4, No. 4 (Septiembre-Diciembre), Año 2020. 
Fomenta el desarrollo socioeconómico por su efecto multiplicador.

$>$ Incorpora y alimenta el gasto público.

$>$ Crea empleo directo e indirecto y mejora la distribución de la riqueza.

$>$ Contribuye con el registro de exportaciones y con el reconocimiento del consumo derivado.

> Aumenta el intercambio comercial y la certidumbre económica.

$>$ Diversifica el patrón productivo al complementar las actividades tradicionales.

Beni (1997) sostiene que el desarrollo regional y el planeamiento turístico integrado deben estar conformados por directrices generales basadas en necesidades identificadas de un área determinada, con orientaciones específicas que tiendan a la gestión y desarrollo permanente del territorio.

Es un proceso continuo, permanente y dinámico con una gran interdependencia de los elementos que lo componen, destinado a establecer los objetivos generales del desarrollo, así como el uso, evaluación y jerarquización de los recursos turísticos. El enfoque del desarrollo económico local busca difundir el desarrollo desde abajo y con los actores locales, tratando de endogeneizar territorialmente las bases de sustentación del crecimiento económico y el empleo productivo. Con ello, además, se incrementan las posibilidades de aprovechar las oportunidades de dinamismo externo existentes (Gambarota \& Lorda, 2017).

Resulta clave la cuestión de la endogeneidad del proceso de desarrollo cuando se aplica esta teoría al campo del turismo debido a que:

1. El desarrollo turístico genera ingresos y empleos; lo que en ocasiones se dificulta es que estos ingresos y empleos se traduzcan en mejoras en la calidad de vida de las comunidades receptoras.

2. Lo anterior no significa adoptar una postura extrema de rechazo a las inversiones extranjeras o extra locales, es importante conocer que se deben aprovechar las oportunidades, pero manteniendo la capacidad de decisión y liderazgo local.

Vázquez Barquero (2000) sostiene que se puede definir el desarrollo económico local como un proceso de crecimiento y cambio estructural, que mediante la utilización del potencial de desarrollo existente en el territorio, conduce a la mejora del bienestar de la población de una localidad o una región. Cuando la comunidad local es capaz de liderar el proceso de cambio estructural, la forma de desarrollo se puede denominarla desarrollo local endógeno. En este contexto, los determinantes del desarrollo endógeno son la creación y difusión de innovaciones, la organización flexible de la producción, el desarrollo urbano del territorio y el desarrollo de las instituciones. 


\section{Los ODS del turismo para una estrategia al desarrollo local y sostenible}

La relación entre los Objetivos de Desarrollo Sostenible (ODS) y el turismo se está definiendo a través de diversos enfoques, considerando importantes sólo aquellos objetivos que se relacionan directamente con el sector, como el de empleo, producción y consumo sostenible, conservación de ecosistemas marinos y terrestres. Por otra parte el turismo rural comunitario es empleado por Agencias de desarrollo y ONG como una estrategia, así como por el sector público local en cuanto a la gestión del territorio, los recursos y el patrimonio, mientras tanto, la implementación de una ruta turística contribuye directa o indirectamente a todos estos objetivos y el turismo sostenible está firmemente posicionado en la agenda para el desarrollo post-2015 y para cumplir aquello es necesario un marco de implementación claro (Gamez, s. f.). A continuación se detallan los ODS que se relacionan con el turismo como estrategia al desarrollo local.

El ODS 1 menciona «Poner fin a la pobreza en todas sus formas en todo el mundo» considerando la meta 1.1, hace referencia que para el 2030 se erradicara la pobreza extrema para todas las personas en el mundo, así el avance en la agenda, se establece a partir de la focalización de programas sociales, con especial atención en la población pobre y vulnerable, por su parte la contribución de aquello puede ser muy alta, porque la implementación de una ruta con alto flujo turístico es una forma de marcar el territorio de los habitantes a beneficio de todos.

El ODS 5 «Lograr la igualdad entre los géneros y empoderar a todas las mujeres y las niñas» para la meta 5.5, hace mención a velar por la participación plena y efectiva de las mujeres y la igualdad de oportunidades de liderazgo a todos los niveles de la adopción de decisiones en la vida política, económica y pública, considerando que para el avance en la agenda se planea mayores oportunidades para las mujeres a través de medidas que incentiven y fortalezcan este ODS, de esta forma todos tendrían diversas oportunidades de superación. Se deben promover medidas para garantizar los mismos derechos y oportunidades laborales a la mujer, por un lado es beneficioso para sus propias familias pues la mujer adquiere un protagonismo porque ya es dueña de lo hace, tiene sus propios ingresos y puede participar en sustentar a su familia. Por otro lado, es beneficioso para la comunidad, como gestora de un emprendimiento turístico y generadora de desarrollo, puede participar en las decisiones de la comunidad con su propia voz, con su propio posicionamiento.

El ODS 8 «Crecimiento económico sostenido, inclusivo y sostenible, el empleo pleno y productivo y el trabajo decente para todos» dentro de la meta 1.5 se propone elaborar y poner en práctica políticas encaminadas a promover un turismo sostenible, que cree puestos de trabajo y promueva la cultura y los productos locales, así en la agenda se aborda el tema de acceso al trabajo en condiciones de igualdad con la finalidad de lograr el empoderamiento económico de las mujeres y promover su autonomía, con propuestas como la reducción de la carga de trabajo no remunerado a través de la ampliación de la cobertura de los servicios de cuidado. Propuestas de acciones para promover el empleo pleno y productivo, además del acceso a créditos para el sector productivo y priorización de la comercialización. Por ello es importante crear en base de hacer un cambio y es un buen inicio para un desarrollo sostenible mediante la implementación de una ruta turística, siendo una gran oportunidad a partir de la productividad que surge a raíz de esto. 
El ODS 12 «Garantizar modalidades de consumo y producción sostenibles» la meta 12.b se basa en elaborar y aplicar instrumentos que permitan seguir de cerca los efectos en el desarrollo sostenible con miras a lograr un turismo sostenible que cree puestos de trabajo y promueva la cultura y los productos locales. Y en la agenda el propósito es hacer más y mejores cosas con menos recursos a través del incremento de proyectos productivos. En esta medida, el turismo puede contribuir a cambiar patrones de consumo y fomentar que sean responsables y sostenibles. Todas las cadenas de turismo responsable involucran la utilización de recursos gastronómicos y actividades que son auténticos, son recursos propios de la comunidad que contribuyen de alguna forma a la generación de un patrón de consumo sostenible, responsable y autóctono (Gamez, 2018.)

\section{RESULTADOS}

Opinión ciudadana respecto a la implementación de una ruta turística en la Parroquia La Pila. El turismo rural comunitario es cada vez más empleado como una estrategia de desarrollo, así como por el sector público local en cuanto a la gestión del territorio, los recursos y el patrimonio. Al respecto, este representa una oportunidad de generación de ingresos para la parroquia La Pila y a partir de aquello se detallan los resultados con la utilización de una técnica como lo es la encuesta denomina "Una ruta turística camino al desarrollo local" que arrojo la información que se demuestran en los siguientes gráficos, en esta medida es importante mencionar que se recolectaron 215 muestras con un margen de error de 6,4\%.

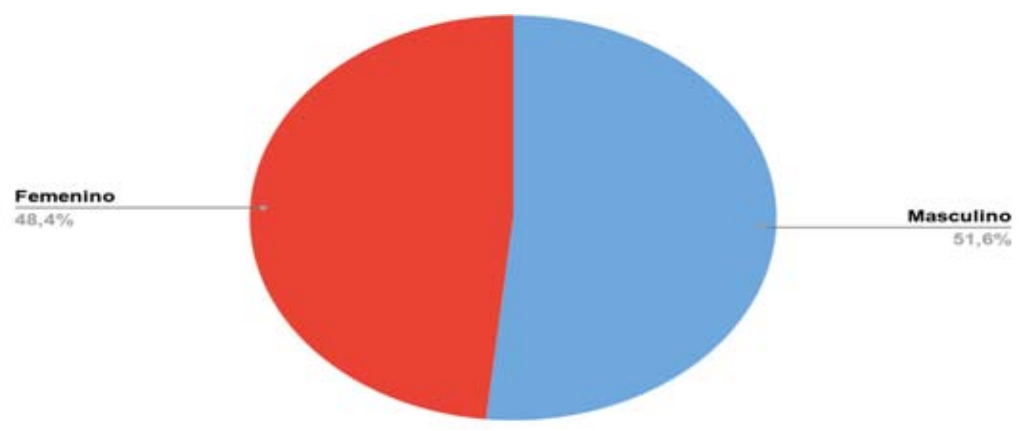

Figura 1. Género de las personas encuestadas

En la encuesta participaron 215 ciudadanos, de los cuales el 51,6 \% son hombre y el 48,4\% son mujeres. 


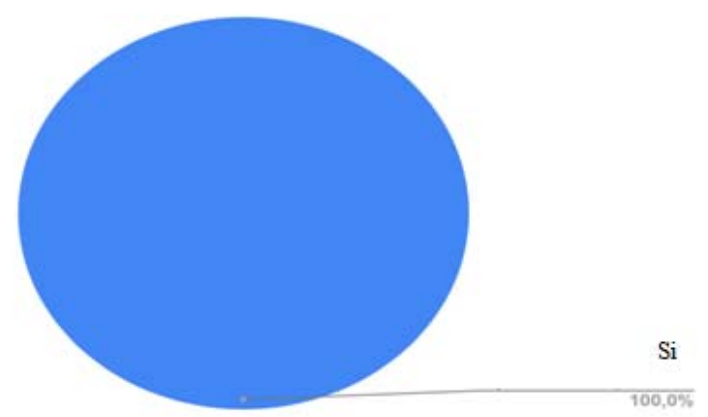

Figura 2. Una ruta turística es una estrategia al desarrollo local para la parroquia La Pila.

En este aspecto el 98,6\% de la comunidad demostró una respuesta positiva, con respecto a la ruta turística como una estrategia, para que la Parroquia La Pila siga el camino para la obtención de un desarrollo local en beneficio de todos a diferencia del 1,4\%, así se deduce que el tema obtuvo una acogida considerable.

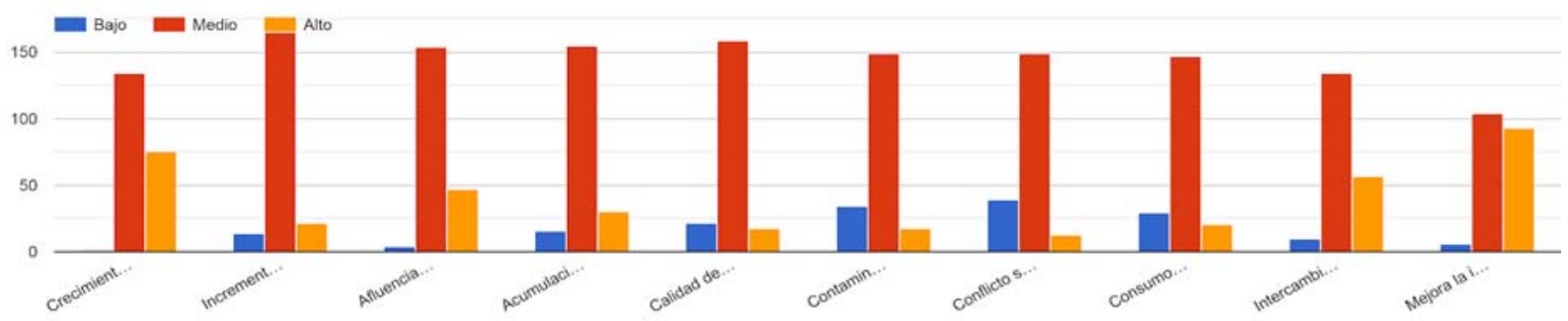

Figura 3: La industria del turismo beneficia a la economía local.

Por medio del gráfico de columnas se detallan diferentes impactos según el nivel de escala que considera cada encuestado, de tal modo es importante mencionarlos: Los impactos y el nivel porcentual se detallan a continuación:

“Crecimiento económico" bajo (0,5\%), medio (63,8\%), alto (35,7; “Incremento de ruido" con un nivel bajo (6,8\%), medio (82,8), alto (10,9\%); “Afluencia vehicular” bajo $(2,0 \%)$, medio $(75,1 \%)$, alto (22,9); “Acumulación de desechos” bajo (8,0\%), medio (77,1\%), alto (14,9\%); "Calidad del agua: bajo (11,1\%), medio (79,9\%), alto (9,0\%); “Contaminación visual” bajo (16,9\%), medio (74,1\%), alto (9,0\%); “Conflicto social” bajo (19,4\%), medio $(74,1 \%)$, alto (6,5\%); "Consumo acelerado de recursos naturales" bajo (14,7\%), medio (74,6\%), alto (10,7\%); "Intercambio cultural entre turistas y residentes" bajo (5,0\%), medio (66,7\%), alto (28,4\%); "Mejora la inversión y la infraestructura física y tecnológica” bajo (3,0\%), medio $(51,2 \%)$, alto $(45,8 \%)$.

Considerando aquello, el color rojo muestra la mayor cantidad de impactos, posterior el color naranja y por último el azul. Lo que demuestra que, la opinión ciudades para aquello se inclina a los impactos a un nivel medio al potenciar el sector turístico. 
El 100\% de los encuestados tuvieron una afinidad a la opción sí, respecto a la pregunta que se muestra en el gráfico, en este sentido consideran que es una oportunidad que cambiaría positivamente su economía.

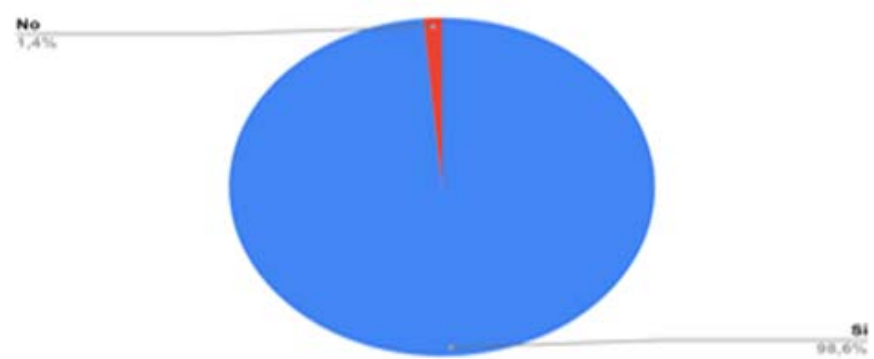

Figura 4. Impacto del sector turístico en la Parroquia La Pila.

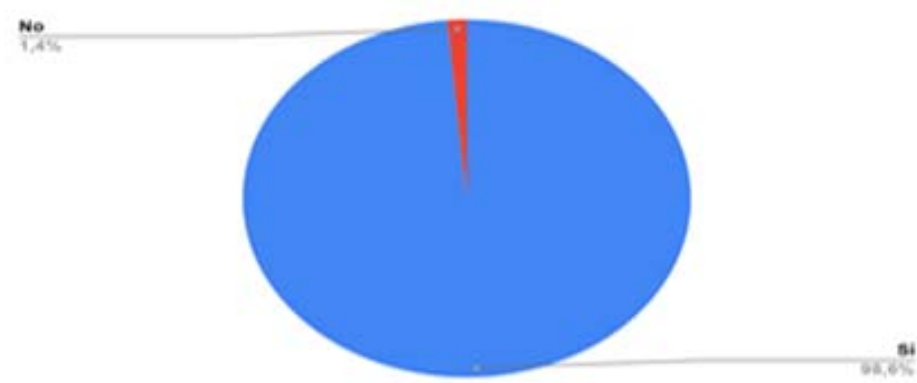

Figura 5. Los ciudadanos formarían parte de programas que incentiven el sector turístico.

La comunidad pílense es caracterizada por ser amantes a situaciones que ayuden al turismo, en este sentido existe motivación por parte del tema y se dedujo que un 92,1\% de encuestados si formarían parte de programas que los motiven a diferencia de un 1,9\%.

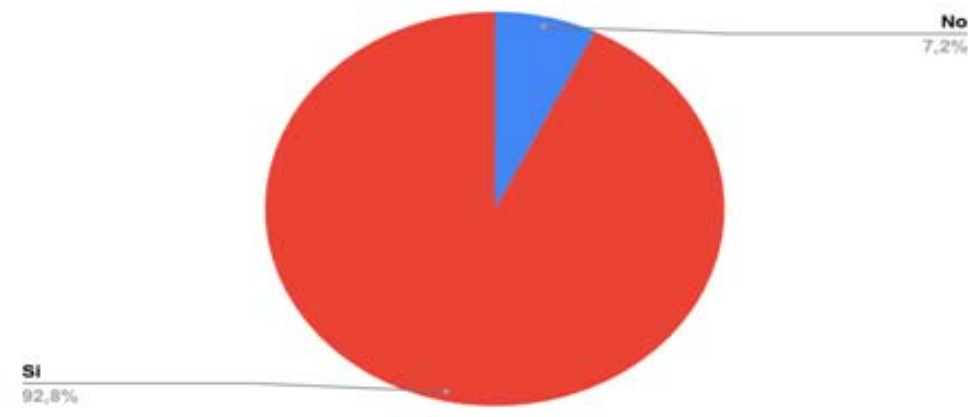

Figura 6. Los habitantes apoyarían propuestas que promuevan la gestión de residuos desde su hogar. 
Considerando este apartado, se obtuvo un resultado positivo porque muestra que el $98,1 \%$ si apoyaría la gestión de residuos desde el hogar, como una buena práctica que beneficia a la parroquia en general a diferencia del 1,9\%.

\section{El desarrollo local generado por la implementación de una ruta turística en La Pila}

Considerando la información del PDOT de la parroquia La Pila y el censo del 2010 según el INEC, de los 2.452 habitantes, existe una población activa liderada por género masculino con un 60,99\% y el género femenino con un 13,85\%; es así que la población inactiva está representada por el género femenino con el 86,15\% frente al género masculino con un 39,01\%; a partir de aquello se evidencia que la población económicamente inactiva supera a la población económicamente activa, lo cual, determina que es necesario aumentar y fortalecer las fuentes de trabajo de acuerdo a la potencialidad del territorio.

Siendo una de las principales actividades el turismo, se propone la creación una ruta turista que ofrece importantes oportunidades de desarrollo sostenible, y los ODS se posicionan en su creación, este es un factor de arrastre que atrae nuevos segmentos al mercado y por su parte, propicia la creación de redes impulsando nuevas fuentes de empleo y negocios, básicamente se restructura la parroquia y se potencia el turismo. Es importante considerar que existe un grupo de artesanos, formado aproximadamente por 200 ciudadanos y son la base para la creación de la ruta turística, según la encuesta realizada se evidencio el interés del tema por parte ellos.

En la rama artesanal, los trabajos en cerámica generan aproximadamente 180 plazas de trabajo, dejando a un lado la artesanía de barros con un índice inferior de empleo, lo que provoca la migración de la población en un $0,49 \%$, dentro de ello se plantea mejorar la comunicación de los habitantes y crear lazos a partir de asociaciones que brinden oportunidades de desarrollo con potencial para la inclusión socioeconómica de la comunidad rural, creando tejido microempresarial y haciendo participe a todos géneros sin discriminación alguna, promoviendo la articulación con otros actores del entorno, por ello es una fuente complementaria de diversificación de ingresos y al participar en el proyecto la parroquia obtendría un crecimiento económico considerable disminuyendo la tasa de migración y en efecto al fortalecer aspectos culturales y sociales se potencia su creación y en relación las condiciones del territorio permite crear espacios para generar el desarrollo local turístico.

A partir de esto, existe interés de parte del gobierno parroquial de implementar la ruta turística y la opinión ciudadana es punto importante a destacar, de acuerdo con los resultados de la encuesta, esta corrobora que participaría en ella, lo que permite que se cumpla con objetivos de desarrollo sostenible considerando su planificación para su posterior ejecución.

\section{CONCLUSIONES}

La actividad turística está en constante crecimiento, consolidándose como una importante vía de ingresos y de gran oportunidad para potenciar un territorio. En efecto la conformación de la ruta turística en la parroquia es una táctica factible y se realiza de la mano de una planificación concertada, con una adecuada gestión y la participación de la población residente que contribuye de forma activa, a fin de que promueve un desarrollo sustentable y contribuye a mejorar su 
calidad de vida. Así su estado actual como estrategia para un desarrollo local ante la opinión ciudadana se determinó por la interpretación de los gráficos que la representa y de forma general se obtuvieron resultado positivo referente al tema, corroborando que los ciudadanos formarían parte de la iniciativa apoyados por gobierno parroquial y conservando sus raíces brindan el servicio requerido para la productividad turística.

Los aportes que se generan al potenciar el turismo en la parroquia La Pila son importantes para lograr un desarrollo local sostenible, al respecto se dedujo que fortalecer aspectos económicos, sociales, ambientales, culturales, patrimoniales, políticos y tecnológicos son puntos claves de acción para lograr los objetivos a fin del beneficio de la ciudadanía, con su implementación se logra ampliar plazas se empleó y se reduce la tasa de migración incrementando la economía y el impacto sostenible de la parroquia en las diferentes actividades realizadas por la ciudadanía y una ampliación en los flujos turísticos para obtener oportunidades de confianza en el mercado.

\section{REFERENCIAS BIBLIOGRÁFICAS}

Abellán, F. C., Ruiz, M. del C. C., Climent, M. P. S., Abellán, A. C., Rubio, M. V., Fernández, D. M., Martín, J. C., Querol, M. de los Á., Boado, F. C., Fernández, J. I. P., Merino, B. R., Romero, R. L., Sarasa, J. L. A., Marín, C. E., Baidal, J. A. I., Cabrera, A. G., Capdepón, F. P., Espín, P. M., Clavé, S. A., ... Balbellido, S. S. (2008). Turismo rural y desarrollo local. Univ de Castilla La Mancha.

BENI, M. C. 1997. «Política e Estrategia de Desenvolvimento Regional. Planejamento Integrado do Turismo». En: A. BALASTRERI RODRÍGUES. (Org.), Turismo e Desenvolvimiento Local, (Vol. 1). pp. 79-86. Hucitec. São Paulo, Brasil.

Cara, R. B. (s. f.). TEORIA DE LA ACCION TERRITORIAL. ACCION TURISTICA Y DESARROLLO. 19.

Coraggio, José Luis, Las políticas públicas participativas: ¿obstáculo o requisito para el desarrollo local? Desarrollo local, Una revisión crítica del debate, Argentina, 2006.

Gambarota, D. M., \& Lorda, M. A. (2017). Como estrategia de desarrollo local. 15.

Gamez, M. J. (2018). Objetivos y metas de desarrollo sostenible. Desarrollo Sostenible. Recuperado 7 de agosto de 2020, de https://www.un.org/sustainabledevelopment/es/objetivos-de-desarrollo-sostenible/

García Ferrando M. La encuesta. En: Garcia M, Ibáñez J, Alvira F. El análisis de la realidad social. Métodos y técnicas de Investigación. Madrid: Alianza Universidad Textos, 1993; p.141-70.

Lopez, E. (2018). METODOLOGIA CUANTITATIVA. https://www.eumed.net/tesisdoctorales/2012/eal/metodologia_cuantitativa.html

Lecanda, R. Q., \& Garrido, C. C. (s. f.). Introducción a la metodología de investigación cualitativa. 36.

MANTERO, Juan Carlos (2004) Desarrollo Local y Actividad Turística. En Aportes y Transferencias, Centro deInvestigaciones Turísticas. UNMdP, año 8 volumen 1; pp. 11 - 38.

MÉNDEZ, R. 1997. Geografía económica. Ariel. Barcelona, España. ORGANIZACIÓN MUNDIAL DE TURISMO. 2003. Turismo y atenuación de la pobreza. Organización Mundial de Turismo. Madrid, España

Periódico Verde. (2011, Enero 14). Turismo Sostenible: Una Estrategia de Desarrollo Local, Regional y Nacional. ECOticias.com. https://www.ecoticias.com/sostenibilidad/40273/Turismo-Sostenible-Una-Estrategia-deDesarrollo-Local-Regional-y-Nacional

(C) Universidad Estatal del Sur de Manabí. Jipijapa, Ecuador. 
Proaño Lucero, G. E. (s. f.). “GESTIÓN Y DESARROLLO TURÍSTICO EN EL CANTÓN GUAMOTE, PROVINCIA $D E$ CHIMBORAZO”.

http://dspace.unach.edu.ec/bitstream/51000/5827/4/Art\%C3\%ADculo\%20t\%C3\%A9cnico\%20005.pdf

Rodríguez Peñuelas, M. A. (2010). Métodos de investigación. 1ra. Edición, México. Ed. Universidad Autónoma de Sinaloa.

Schulte, Silke: Desarrollo del turismo Sostenible: ¿Una Utopía?: Tesis para Magister en Asentamientos Humanos y Medio Ambiente, Pontificia Universidad Católica Santiago de Chile, 2002

Varisco, C. (2008). Desarrollo Turístico y Desarrollo Local. 208.

VÁZQUEZ BARQUERO, A. 2001. «Desarrollo endógeno y globalización». En: A. VÁZQUEZ BARQUERO y O. MADOERY (Compiladores), Transformaciones globales, instituciones y políticas de desarrollo local. pp. 7699. Homo Sapiens, Rosario, Santa Fe.

VERA REBOLLO, J. F. (coord.); LÓPEZ PALOMEQUE, F.; MARCHENA GÓMEZ, M.; ANTÓN, S.; prólogo de VILA VALENTÍ, J. 1997. Análisis territoriales del turismo. Una nueva Geografía del Turismo. Ariel, Barcelona, España.

Xercavins, J., Cayuela, D., Cervantes, G., \& Sabater, A. (2005). En Desarrollo Sostenible. Barcelona: Ediciones Graficas Rey

192 UNESUM-Ciencias. Publicación cuatrimestral. Vol. 4, No. 4 (Septiembre-Diciembre), Año 2020. 